\title{
PERMEABILIDADE DE HIDROGÊNIO EM AÇO ALTA RESISTÊNCIA BAIXA LIGA GRAU X80 CONTENDO NIÓBIO*
}

\author{
Duberney Hincapié-Ladino ${ }^{1}$ \\ Samar de Carvalho Lopes da Silva² \\ José Wilmar Calderón-Hernandez \\ Neusa Alonso Falleiros ${ }^{4}$
}

\section{Resumo}

O presente trabalho estuda os parâmetros de permeabilidade de hidrogênio de um tubo API 5L X80, e uma chapa de aço grau X80, onde a principal diferença está na composição química, o primeiro contém $0,07 \% \mathrm{C}$ e $0,04 \% \mathrm{Nb}$ e o segundo $0,04 \% \mathrm{C}$ e $0,102 \% \mathrm{Nb}$. As amostras foram cortadas com espessura de aproximadamente $1 \mathrm{~mm}$, lixadas e recobertas com um filme de níquel, em uma das faces. Nos ensaios de permeabilidade foi utilizada uma célula modificada tipo Devanathan-Stachurski, com a solução A da norma NACE TM0284, com injeção de $\mathrm{H}_{2} \mathrm{~S}$ no lado de geração e $0,2 \mathrm{M}$ $\mathrm{NaOH}$ no lado de detecção. As curvas de corrente em função do tempo obtidas passaram por tratamentos matemáticos (método $t_{\text {lag }}$ ), permitindo a obtenção dos parâmetros de difusividade aparente, concentração máxima de hidrogênio e número de traps (sítios de ancoramento). Tais parâmetros mostraram-se iguais para os dois materiais. As curvas foram normalizadas e modificadas de maneira a eliminar o efeito da espessura das amostras. Estas novas curvas foram comparadas com o modelo teórico da segunda lei de Fick. A comparação evidenciou que o procedimento inicial para a obtenção dos parâmetros de permeabilidade mostra-se eficiente e vantajoso por apresentar maior praticidade. Pôde-se concluir que a semelhança no comportamento dos parâmetros de permeabilidade foi devida às similaridades nas suas microestruturas. Os resultados obtidos no presente trabalho evidenciam que o tipo e quantidade de traps nestes materiais são determinados pela microestrutura.

Palavras-chave: Permeabilidade de hidrogênio, Difusividade de hidrogênio; Sítio de ancoramento; Nióbio.

\section{HYDROGEN PERMEATION IN X80 MICROALOYED STEEL WITH HIGH NIOBIUM CONTENTS}

\section{Abstract}

This paper study the hydrogen permeability parameters of API $5 \mathrm{~L}$ X80 pipeline steel, and a plate grade $\mathrm{X} 80$, the main difference is the chemical composition, $0.07 \% \mathrm{C}$ and $0.04 \% \mathrm{Nb}$ and $0.04 \% \mathrm{C}$ and $0.102 \% \mathrm{Nb}$, respectively, specimens were cut to be approximately $1 \mathrm{~mm}$ thickness, sanded and nickel plated in one side. A modified Devanathan's cell was used in the experiments. The hydrogen charge compartment was filled with NACE solution A with $\mathrm{H}_{2} \mathrm{~S}$ injections and the oxidation compartment was filled with $0.2 \mathrm{M} \mathrm{NaOH}$ solution, A timecurrent characteristic curve was obtained, time-lag method was used to determine apparent diffusivity, hydrogen concentration and the number of trap sites. These parameters were the same for both materials. All the curves were normalized to eliminate the effect of sample thickness. These new curves were compared with the theoretical model of the Fick's second law. Time-lag method was showed advantages than the curve-fitting method for determine the permeation parameters. It was concluded that the similarity in the permeability parameters was due to similarities in their microstructures. The results obtained in this study showed that the type and amount of traps sites were determined by the microstructure.

Keywords: Hydrogen permeation; Hydrogen diffusivity, Hydrogen trap site; Niobium.

1 Doutorando, Departamento de Engenharia Metalúrgica e de Materiais, Universidade de São Paulo (USP), São Paulo, Brasil.

2 Engenheira, Departamento de Engenharia Metalúrgica e de Materiais, USP, São Paulo, Brasil.

3 Doutor, Departamento de Engenharia Metalúrgica e de Materiais, USP, São Paulo, Brasil.

4 Doutora, Departamento de Engenharia Metalúrgica e de Materiais, USP, São Paulo, Brasil. 


\section{INTRODUÇÃO}

A permeabilidade do hidrogênio nos aços tem um papel central no estudo da fragilização e trincamento induzido pelo hidrogênio (HIC). O hidrogênio adsorvido na superfície do material é parcialmente retornado à atmosfera na forma de $\mathrm{H}_{2}$ e parcialmente difundido e absorvido no material, se localizando em lugares preferenciais. A permeabilidade do hidrogênio está relacionada com esta quantidade de hidrogênio difundido pelo material e permite a derivação da quantidade de hidrogênio aprisionada no material (traps). Tais medidas são de suma importância pois dimensionam a capacidade do material armazenar hidrogênio que pode levar a possíveis falhas. Diversos parâmetros podem afetar a permeabilidade de hidrogênio. Entre eles está a pressão parcial do $\mathrm{H}_{2} \mathrm{~S}$ ( $\mathrm{P}_{\mathrm{H} 2 \mathrm{~S}}$ ) e pH da solução. Estudos, como o de Zhou et al. (2013) [1], mostram que a permeabilidade de $\mathrm{H}$ aumenta com o aumento da $\mathrm{P}_{\mathrm{H} 2 \mathrm{~S}}$, devido à maior formação de hidrogênio na superfície do metal, e diminui com o aumento do $\mathrm{pH}$. Pode ser definida uma permeabilidade crítica do hidrogênio para a ocorrência das falhas, que depende da composição química e microestrutura do aço $[1,2]$.

A microestrutura apresenta uma elevada influência na permeabilidade de hidrogênio. Um estudo comparativo entre a permeabilidade de $\mathrm{H}$ e as diferentes microestruturas, mostra que a ferrita acicular possui menor permeabilidade de hidrogênio, que se deve à maior quantidade de traps reversíveis na estrutura, devido à maior quantidade de interfaces causada pelos grãos não orientados de ferrita acicular [3]. Porém, outras análises mostraram que a alta tenacidade da ferrita acicular dificulta a propagação das trincas, enquanto na fase bainítica, a distribuição de aglomerados martensíticos promove uma maior susceptibilidade ao HIC [1, 2].

Diversas investigações já foram realizadas com o uso da permeabilidade de hidrogênio obtida pela técnica eletroquímica. Em 2011, Huang et al. [4] estudaram a influência de tratamentos térmicos e suas respectivas microestruturas em um aço X80 com $0,041 \%$ C e $0,095 \% \mathrm{Nb}$, comparando as microestruturas das amostras original, aquecida a $1300^{\circ} \mathrm{C}$ e resfriada ao ar e em água e suas eficiências de ancoramento do hidrogênio, bem como suas suscetibilidades ao HIC. A amostra original apresentou uma estrutura de ferrita acicular com martensita dispersa, a resfriada ao ar apresentou ferrita poligonal com grãos de bainita e a resfriada em água apresentou martensita e austenita retida. Os testes de resistência ao HIC mostraram que as amostras com tratamento térmico apresentaram mais suscetibilidade ao trincamento do que a amostra original; além disso, os resultados mostraram que trincas se iniciaram em inclusões de $\mathrm{Mn}$ e $\mathrm{Al}$, enquanto que inclusões de Si não apresentaram trincas. Huang et al. (2011) [4] mostraram que quanto maior a difusividade aparente e a permeabilidade do hidrogênio, menor é a solubilidade aparente do hidrogênio no aço, isto é, menos átomos de hidrogênio são ancorados e portanto menor a susceptibilidade ao $\mathrm{HIC}$, o que foi comprovado pelos autores no ensaio de HIC.

As inclusões podem ou não ser sítios de elevada eficiência de ancoramento de hidrogênio, portanto influenciando também na susceptibilidade a HIC. Em 2009, Dong et al. [5] estudaram as inclusões em aços X70, cuja microestrutura era ferrita poligonal com uma fração menor de ferrita acicular. Os resultados mostraram que nitretos de titânio foram os principais sítios de início de trinca, além disso, trincas também foram observadas nos contornos de grão da ferrita poligonal, porém não nos contornos de grão da ferrita acicular, o que é consistente com os resultados encontrados por Park et al., em 2008, [3] que mostraram que a ferrita acicular não é 
um sítio favorável de ancoramento de hidrogênio. Em 2011, Xue et al. [6] fizeram um estudo similar sobre inclusões em um aço X80 com $0,078 \% \mathrm{Nb}$, relacionando a suscetibilidade a HIC com a permeabilidade, onde foram encontradas trincas iniciadas em inclusões de óxidos de Si e Al. Porém, inclusões ricas em carboneto de ferro e uma mistura de Al-Mg-Ca-O não foram locais de início de trincas.

$\mathrm{Na}$ fabricação de tubulações, a soldagem também é um importante processo, pois a zona de soldagem possui diferente composição química e microestrutura, apresentando, portanto, uma diferente susceptibilidade ao HIC do que do o restante do componente metálico. Em 2003, Wang et al. [7] analisaram a permeabilidade de hidrogênio em aços soldados por arco submerso. O metal base, com microestrutura de ferrita com fase dispersa de perlita, apresentou alta permeabilidade enquanto a zona termicamente afetada (ZAC), com microestrutura bainítica, e o metal de solda, que apresenta zonas de martensita e austenita retida, apresentaram permeabilidades cada vez mais baixas. Ou seja, a soldagem afeta a susceptibilidade a trinca induzida por hidrogênio de forma que o metal de solda apresenta sítios mais favoráveis para a nucleação e propagação de trinca, seguido da ZAC e do metal base.

\section{MATERIAIS E MÉTODOS}

Para realização dos ensaios foram utilizadas amostras denominadas de X80 $0,1 \mathrm{Nb}$ (placa) e X80 (tubo comercial), disponíveis no Laboratório de Processos Eletroquímicos (LPE) da USP. As composições químicas das amostras disponíveis podem ser observadas na Tabela 1.

Tabela 1. Composição química das amostras, porcentagem em massa

\begin{tabular}{|c|c|c|c|c|c|c|c|c|c|c|c|}
\cline { 2 - 9 } & $\mathbf{C}$ & $\mathbf{M n}$ & $\mathbf{S i}$ & $\mathbf{P}$ & $\mathbf{S}$ & $\mathbf{C u}$ & $\mathbf{T i}$ & $\mathbf{V}$ & $\mathbf{N b}$ & $\mathbf{M o}$ & $\mathbf{C r}$ \\
\hline X80 0,1 Nb & $\mathbf{0 , 0 4}$ & 1,78 & 0,21 & 0,009 & 0,001 & 0,200 & 0,012 & 0,001 & $\mathbf{0 , 1 0 2}$ & 0,02 & 0,36 \\
\hline X80 & $\mathbf{0 , 0 7}$ & 1,79 & 0,33 & 0,012 & 0,001 & 0,013 & 0,013 & 0,004 & $\mathbf{0 , 0 4}$ & 0,13 & 0,16 \\
\hline
\end{tabular}

\subsection{Caracterização microestrutural}

Para a caracterização das inclusões foram retiradas amostras no sentido longitudinal tomando-se toda a espessura, foram embutidas em baquelite e polidas até pasta de diamante $1 \mu \mathrm{m}$. Neste ponto foram examinadas em microscópio óptico (MO) e microscópio eletrônico de varredura (MEV), a fim de se caracterizar as inclusões e realizar análises por espectroscopia de energia dispersiva (EDS). Para a caracterização da microestrutura foram retiradas amostras de cada tipo de aço na seção transversal tomando-se, igualmente, toda a espessura, que foram embutidas em baquelite e polidas até pasta de diamante $1 \mu \mathrm{m}$ e atacadas com nital $2 \% \mathrm{e}$ examinadas em MEV.

\subsection{Ensaios de permeabilidade de hidrogênio}

Para a realização dos ensaios de permeabilidade de hidrogênio foram cortadas amostras com dimensões de $20 \mathrm{~mm} \times 20 \mathrm{~mm}$ aproximadamente com espessura de $1 \mathrm{~mm}$. Uma das faces dos corpos de prova foi lixada até lixa 1200 e passaram por processo de niquelação (face da detecção de hidrogênio atômico), sendo a outra 
face lixada até lixa 600 (geração de hidrogênio atômico), lembrando que a face exposta aos eletrólitos é a face transversal ao sentido de laminação.

Foi utilizada uma célula tipo Devanathan-Stachurski [8], modificada para que no lado de geração de hidrogênio fosse injetado $\mathrm{H}_{2} \mathrm{~S}$. Os eletrólitos utilizados foram, no lado de geração de hidrogênio $5 \% \mathrm{NaCl}+0,5 \% \mathrm{CH}_{3} \mathrm{COOH}$ (solução $\mathrm{A}$ ) e do lado de detecção de hidrogênio $0,2 \mathrm{M} \mathrm{NaOH}$. As soluções foram desaeradas em recipientes separados, com injeção de $\mathrm{N}_{2}$ durante 1 hora antes de serem introduzidas na célula. Foi utilizado um potenciostato PAR 273A da Princeton Applied Research, controlado pelo programa PowerCORR - 2.47, Princeton Applied Research (Copyright 2003), com eletrodo de referência de $\mathrm{Ag} / \mathrm{AgCl}$ e um contra-eletrodo de platina.

O eletrólito de $0,2 \mathrm{M} \mathrm{NaOH}$ foi transferido à célula (lado da detecção) até cobrir completamente a amostra, e então foi realizada a aplicação de potencial de eletrodo de $200 \mathrm{mV}(\mathrm{Ag} / \mathrm{AgCl})$, que tem por objetivo retirar o máximo teor de hidrogênio presente no metal. A densidade de corrente era registrada em função do tempo até que fossem atingidos valores estáveis da ordem de $1 \mu \mathrm{A} \cdot \mathrm{cm}^{-2}$. Após o decaimento, a polarização era interrompida. Seguidamente, fazia-se a injeção da solução $A$, no lado de geração de hidrogênio, previamente desaerada e, quando a amostra era totalmente coberta, iniciava-se a injeção de $\mathrm{H}_{2} \mathrm{~S}$. Novamente era aplicado um potencial de eletrodo de $200 \mathrm{mV}(\mathrm{Ag} / \mathrm{AgCl})$, e se iniciava o registro da corrente em função do tempo.

\section{RESULTADOS E DISCUSSÃO}

\subsection{Análise das inclusões e microestrutura}

A Figura 1 apresenta as micrografias em $\mathrm{MO}$ das amostras sem ataque com aumento de 100X, evidenciando as inclusões da superfície, podendo-se dizer que os dois aços têm um nível similar de inclusões.

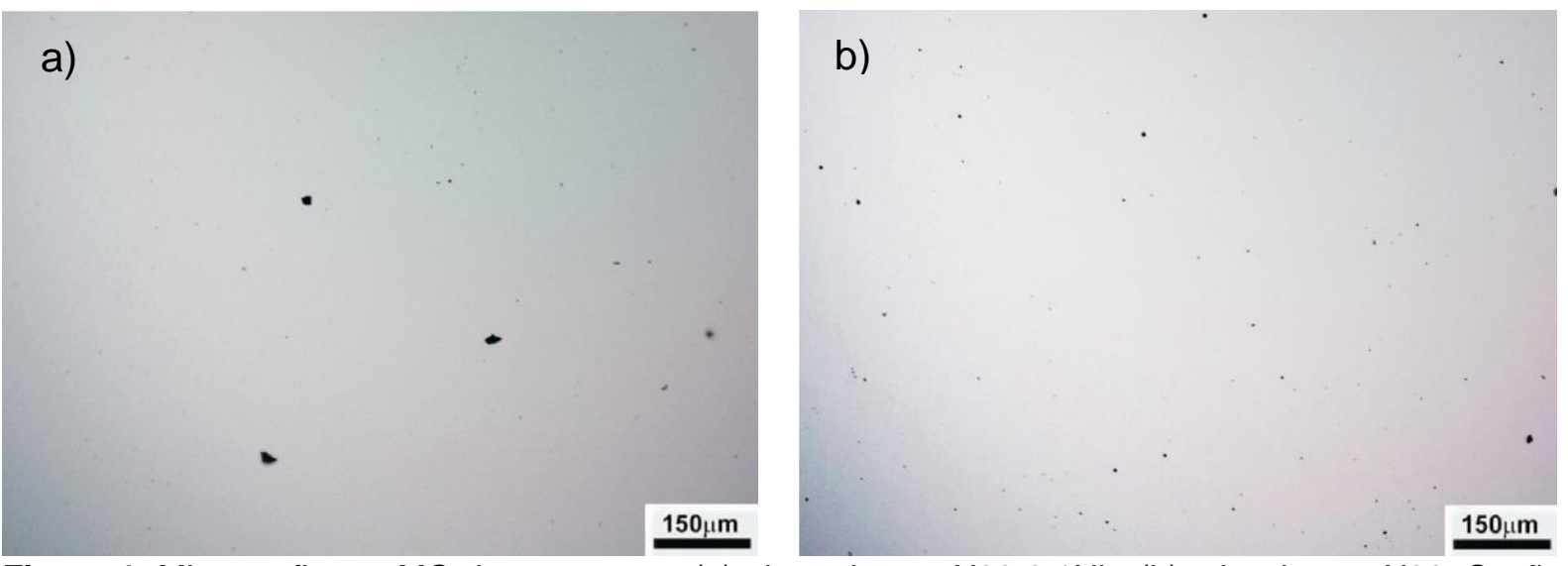

Figura 1. Micrografia em MO das amostras: (a) placa de aço X80 0,1Nb; (b) tubo de aço X80. Seção longitudinal. Polimento $1 \mu \mathrm{m}$, sem ataque. Aumento $100 \mathrm{X}$.

Quando levada ao MEV a amostra X80 $0,1 \mathrm{Nb}$ apresentou dois tipos de inclusões. $\mathrm{Na}$ imagem da Figura $2 \mathrm{a}$ pode-se observar uma inclusão arredondada, com tamanho não maior que $2 \mu \mathrm{m}$. A análise por EDS, mostrou (Figura 2) principalmente a presença de $\mathrm{Mg}$, Ti e $\mathrm{Nb}$. Além desse tipo de inclusões, foram também observadas inclusões com formato alongado com aproximadamente $40 \mu \mathrm{m}$ de comprimento (Figura 3a). A análise por EDS dessa inclusão mostrou presença de $\mathrm{Ca}, \mathrm{Al}, \mathrm{Mg}, \mathrm{Si}$ e S, como apresentado na Figura 3b. 
1

a)
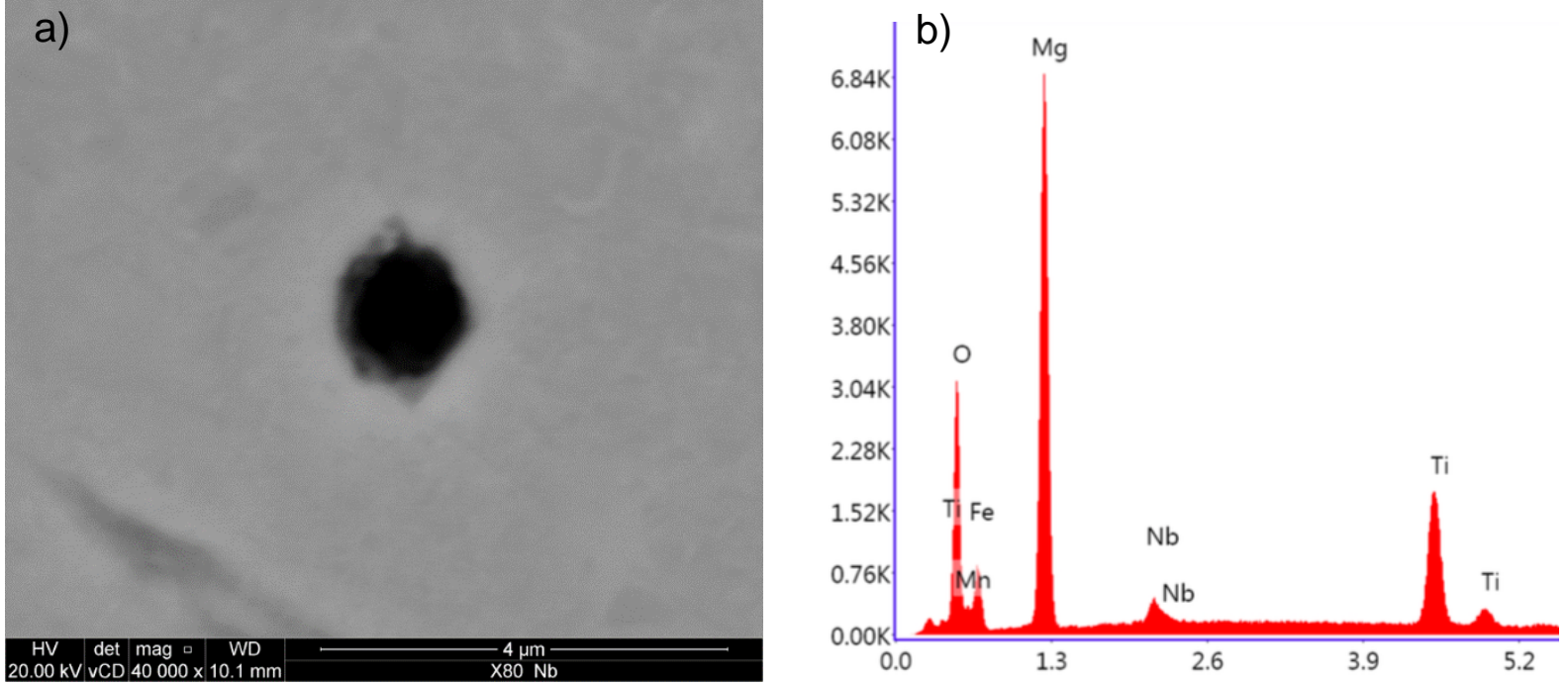

Figura 2. Placa X80 0,1 Nb: (a) inclusão com formato arredondo; (b) EDS: destacam-se Mg, Ti e Nb. Seção longitudinal. Sem ataque. MEV. Elétrons retroespalhados. Aumento de 40.000X.
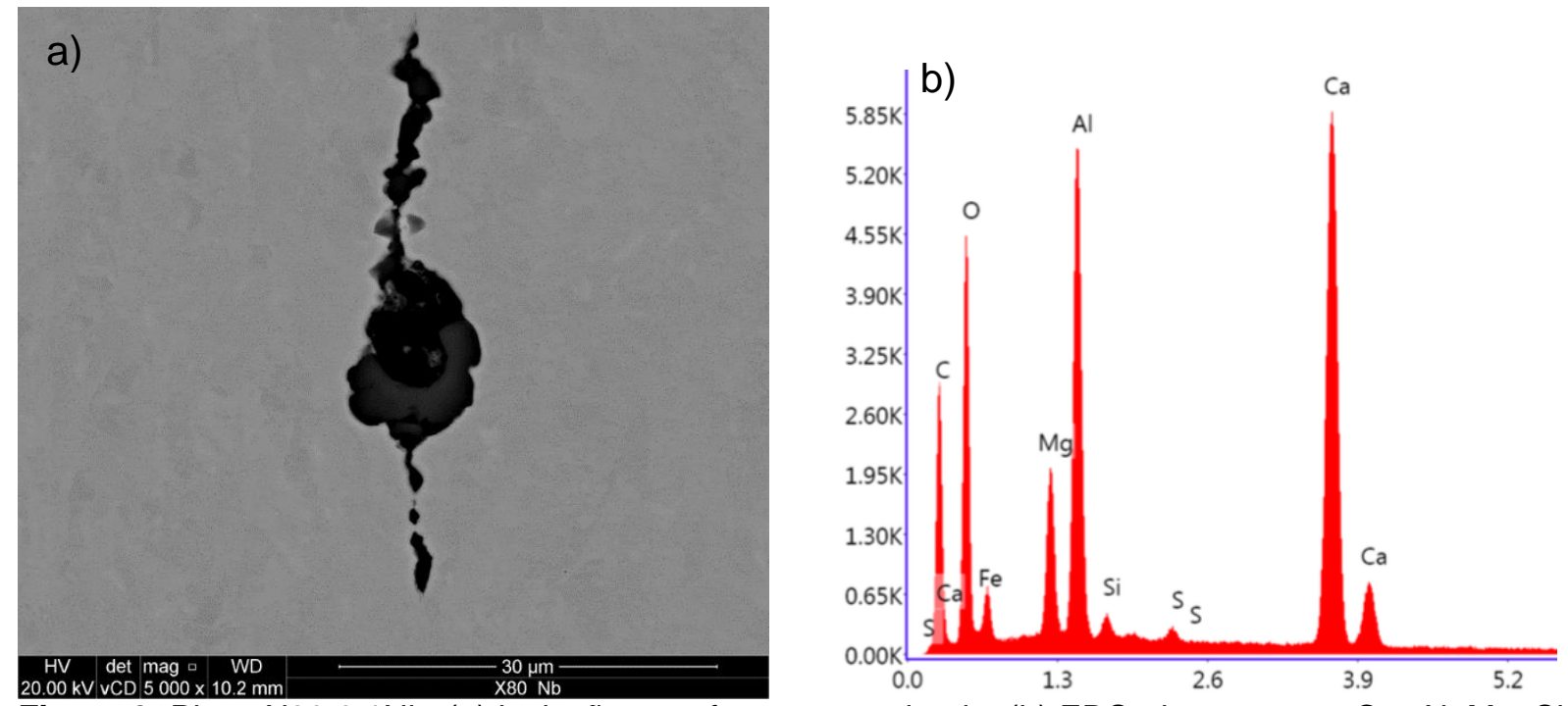

Figura 3. Placa X80 0,1 Nb: (a) inclusão com formato arredondo; (b) EDS: destacam-se $\mathrm{Ca}, \mathrm{Al}, \mathrm{Mg}, \mathrm{Si}$ e S. Seção longitudinal. Sem ataque. MEV. Elétrons retroespalhados. Aumento de 5.000X.

Da mesma maneira o tubo X80 apresentou dois tipos de inclusões. Inclusões de formato arredondado (Figura 4a), não maiores que $10 \mu \mathrm{m}$. O EDS indicou a presença de $\mathrm{Al}, \mathrm{Mg}$ e Ca (Figura 4b). Foram observadas também inclusões de formato alongado, como apresentado na Figura 5a. Estas inclusões apresentam cerca de $30 \mu \mathrm{m}$ de comprimento e a análise por EDS mostrou a presença de $\mathrm{Al}$ e $\mathrm{Ca}$ (Figura 5b). 

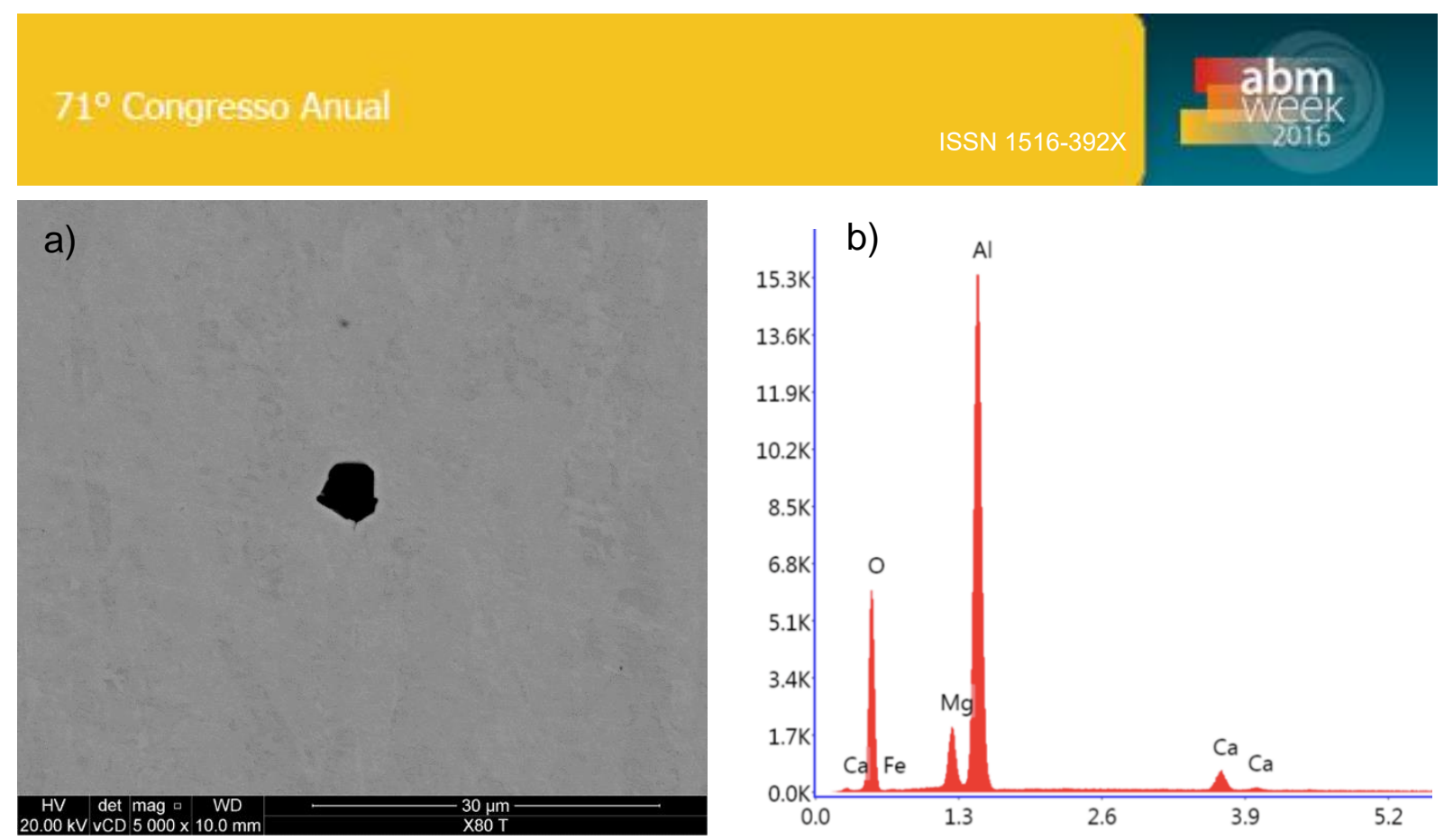

Figura 4. Tubo X80: (a) inclusão com formato arredondo; (b) EDS: Al, Mg e Ca. Seção longitudinal. Sem ataque. MEV. Elétrons retroespalhados. Aumento de 5.000X.
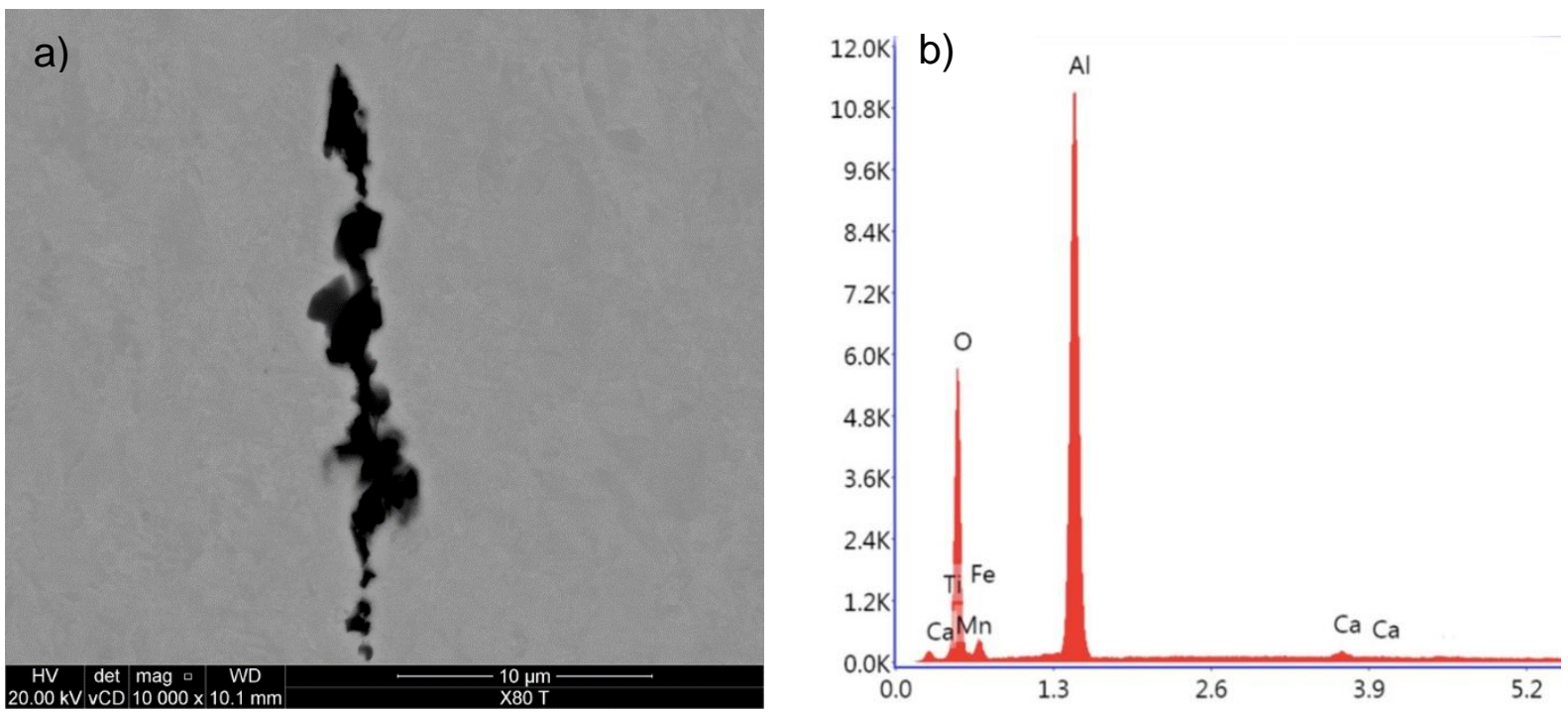

Figura 5. Tubo X80: (a) inclusão com formato alongado; (b) EDS: Al e Ca. Seção longitudinal. Sem ataque. MEV. Elétrons retroespalhados. Aumento de 10.000X.

Nas Figuras 6a e 6b são apresentadas as microestruturas dos materiais X80 0,1 Nb e X80, respectivamente. Em ambos podem ser observadas uma fase acinzentada, caracterizando a matriz ferrítica, e uma fase esbranquiçada em menor quantidade, caracterizando o microconstituinte M/A (martensita/austenita). Comparando-se as micrografias dos dois materiais, observa-se que a microestrutura do tubo X80 é mais refinada do que a da placa $X 80,0,1 \mathrm{Nb}$. Seria esperado que com a adição de $0,1 \%$ $\mathrm{Nb}$, a placa X80 $0,1 \mathrm{Nb}$ apresentasse microestrutura mais refinada, uma vez que o $\mathrm{Nb}$ é um dos principais elementos que promovem o refino de grão em aços ARBL [9]. No entanto, o refino de grão é obtido mediante a adição de microligas e de ciclos de deformação, no campo de estabilidade da austenita. No presente caso, não são conhecidos os processos de conformação mecânica utilizados nos dois materiais aqui estudados. Por isso, não é possível explicar com detalhe a granulação menos refinada observada na placa $X 800,1 \mathrm{Nb}$; provavelmente, isso se deve ao processo de conformação utilizado. 

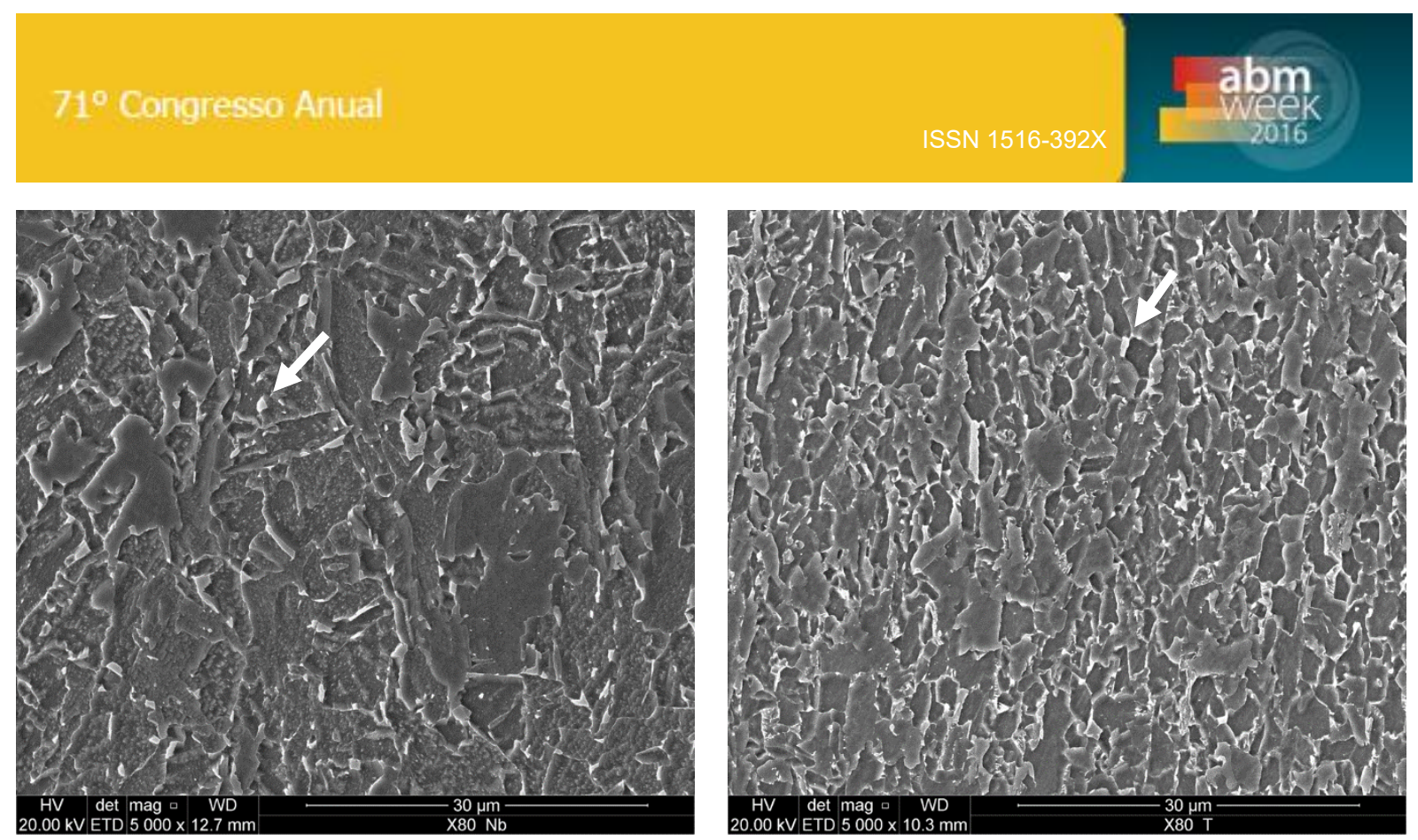

Figura 6. Imagens de elétrons secundários: (a) placa $X 800,1 \mathrm{Nb}$, onde nota-se a presença das fases ferrita e M/A; (b) tubo X80, que apresenta as mesmas fases, ferrita e M/A. Seção transversal. Ataque nital $2 \%$. Aumento de 5.000X. Setas indicando o microconstituinte M/A.

\subsection{Permeabilidade de hidrogênio}

As curvas de permeabilidade de hidrogênio são apresentadas na Figura 7. Com o prosseguimento do ensaio, percebe-se um aumento dos valores de corrente, que representa o aumento da passagem do hidrogênio através do corpo de prova, até atingir o patamar de corrente do regime estacionário, onde o ensaio é finalizado.

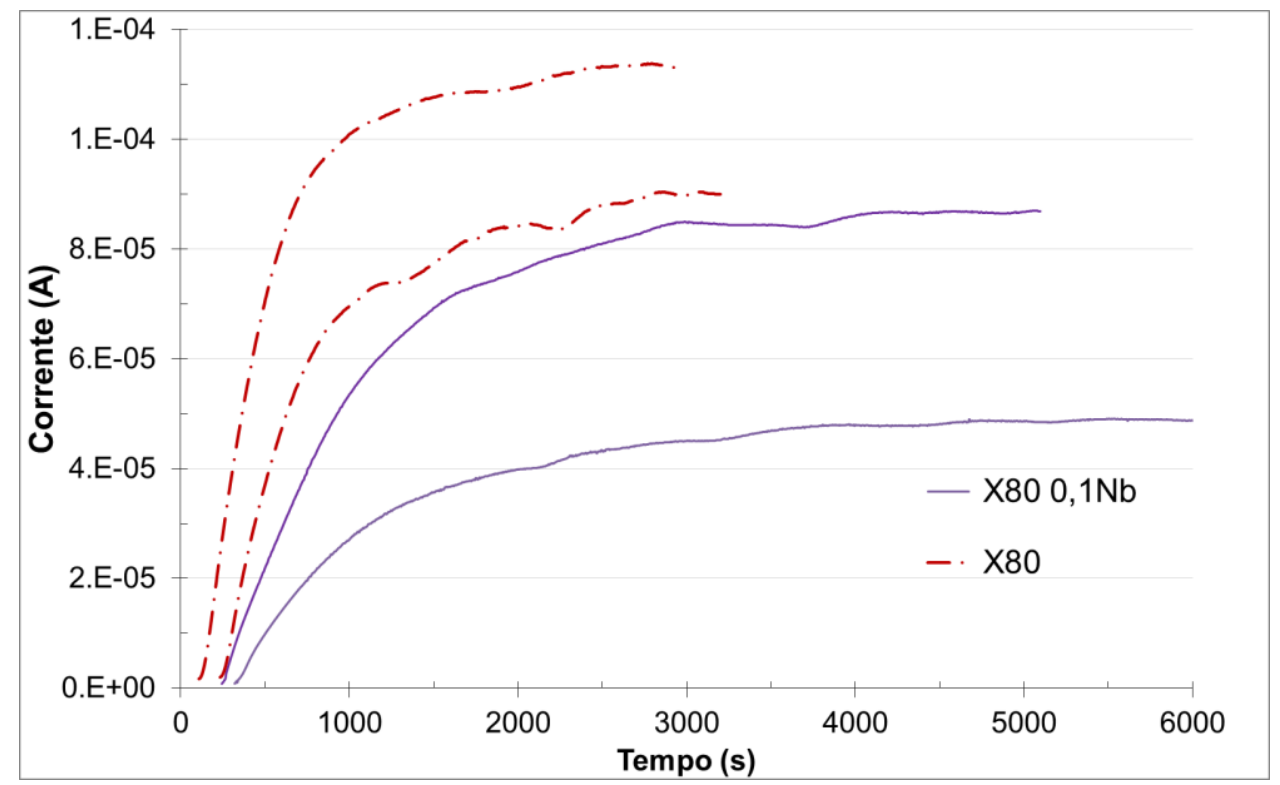

Figura 7. Permeabilidade de hidrogênio para a placa de aço X80 0,1 Nb (linhas cheias) e tubo X80 (linhas tracejadas). Dois ensaios para cada amostra.

$\mathrm{Na}$ Figura 7 nota-se que as curvas para o tubo X80 apresentam um desvio para a esquerda em relação às curvas obtidas para a placa X80 0,1 Nb. Mais adiante, essas curvas passaram por tratamento de normalização, onde essas diferenças foram eliminadas. Lá se discute que a causa da posição diferenciada das curvas está relacionada com as espessuras dos corpos de prova utilizados. 
A partir das curvas obtidas nos ensaios de permeabilidade, podem ser feitos tratamentos matemáticos dos dados [8], de forma a obter-se informações importantes sobre fenômenos que ocorrem devido a passagem do hidrogênio pelos materiais. Para os cálculos dos parâmetros de permeabilidade foi utilizada a metodologia do tlag [8]. Os valores de tlag foram determinados mediante a leitura direta dos gráficos das curvas de permeabilidade de hidrogênio, após ser determinado o valor de corrente de estado estacionário.

Primeiramente foi calculada a difusividade aparente a partir da equação 1 , onde $L$ é a espessura da amostra em centímetros $(\mathrm{cm})$. O tlag pode ser lido diretamente no gráfico já que corresponde ao tempo em segundos necessário para se atingir $63 \%$ da corrente de estado estacionário.

$t_{\operatorname{lag}}=\frac{\mathbf{L}^{2}}{6 D_{\text {eff }}}$

A concentração máxima de hidrogênio atômico $\left(\mathrm{C}_{0}\right)$ presente no aço foi calculada utilizando a equação 2 , onde $\mathrm{J}_{\infty}$ é o fluxo de permeação de hidrogênio do estado estacionário, o qual foi obtido aplicando-se a equação 3 , que utiliza o valor de corrente estacionária ( $\left.\mathrm{I}_{\infty}\right)$. Nessa equação $\mathrm{F}$ representa a constante de Faraday, 96485 C. $\mathrm{mol}^{-1}$, e A representa a área exposta da amostra, 0,8 cm².

$$
\begin{aligned}
& \mathbf{J}_{\infty}=\mathbf{D}_{\text {eff }} \frac{C_{0}}{L} \\
& J_{(t)}=\frac{I_{(t)}}{F A}
\end{aligned}
$$

O número de traps de hidrogênio por unidade de volume $\left(\mathrm{N}_{\mathrm{t}}\right)$ pode ser calculado a partir do $\mathrm{D}_{\text {eff }}$ e da $\mathrm{C}_{0}$, com a equação 4.

$$
\mathbf{N}_{\mathrm{t}}=\frac{\mathrm{C}_{\mathbf{0}}}{3}\left(\frac{\mathrm{D}_{\mathbf{l}}}{\mathrm{D}_{\mathrm{eff}}}-\mathbf{1}\right) \mathbf{N}_{\mathrm{A}}
$$

Nessa equação $D_{1}$ representa o coeficiente de difusão característico do material, sendo utilizado $D_{1}=1,28 \times 10^{-4} \mathrm{~cm}^{2} . \mathrm{s}^{-1}[5,6,10]$ e $\mathrm{N}_{\mathrm{A}}$ é o número de Avogadro. Os parâmetros $D_{\text {eff, }} C_{0}$ e $\mathrm{N}_{\mathrm{t}}$, dos dois materiais examinados no presente trabalho, apresentaram-se praticamente iguais, como apresentado na Tabela 2. Isso mostra que as diferenças de composição química, principalmente os teores de $\mathrm{C}$ e $\mathrm{Nb}$, entre os materiais, não afetam a permeabilidade. Os materiais apresentaram o mesmo comportamento devido à semelhança na microestrutura: ambos apresentam matriz ferrítica com partículas de $\mathrm{M} / \mathrm{A}$. Esses resultados reforçam que a microestrutura é um dos principais responsáveis pelos tipos e quantidade de traps no material.

Tabela 2 Parâmetros calculados a partir do método tlag [8]

\begin{tabular}{|c|c|c|c|c|}
\cline { 2 - 5 } \multicolumn{1}{c|}{} & $\mathbf{t}_{\text {lag }}(\mathbf{s})$ & $\mathbf{D}_{\text {eff }}\left(\mathbf{c m}^{2} . \mathbf{s}^{-1}\right)$ & $\mathbf{C}_{\mathbf{0}}\left(\mathbf{m o l} . \mathbf{c m}^{-2}\right)$ & $\mathbf{N}_{\mathbf{t}}\left(\mathbf{c m}^{-\mathbf{3}}\right)$ \\
\hline \multirow{2}{*}{ X80 0,1 Nb } & 1161 & $1,75 \times 10^{-6}$ & $3,99 \times 10^{-5}$ & $5,75 \times 10^{20}$ \\
\cline { 2 - 5 } & 1021 & $1,90 \times 10^{-6}$ & $6,36 \times 10^{-5}$ & $8,43 \times 10^{20}$ \\
\hline \multirow{2}{*}{ X80 } & 1762 & $1,23 \times 10^{-6}$ & $6,87 \times 10^{-7}$ & $1,42 \times 10^{21}$ \\
\cline { 2 - 5 } & 1599 & $2,27 \times 10^{-6}$ & $5,37 \times 10^{-7}$ & $5,95 \times 10^{20}$ \\
\hline
\end{tabular}


Para melhor comparação entre os resultados obtidos, são apresentadas curvas de permeabilidade normalizadas, portanto, sem a influência da espessura dos corpos de prova (Figura 8). Nota-se que as curvas são muito próximas e que, similarmente ao observado na Tabela 2 , as curvas para a placa X80 $0,1 \mathrm{Nb}$ são praticamente iguais e posicionam-se entre as curvas obtidas para o tubo X80; em todos os casos, as diferenças de inclinação observadas na Figura 7, foram eliminadas. Tais fatos confirmam que os parâmetros de permeabilidade dos dois materiais são praticamente iguais.

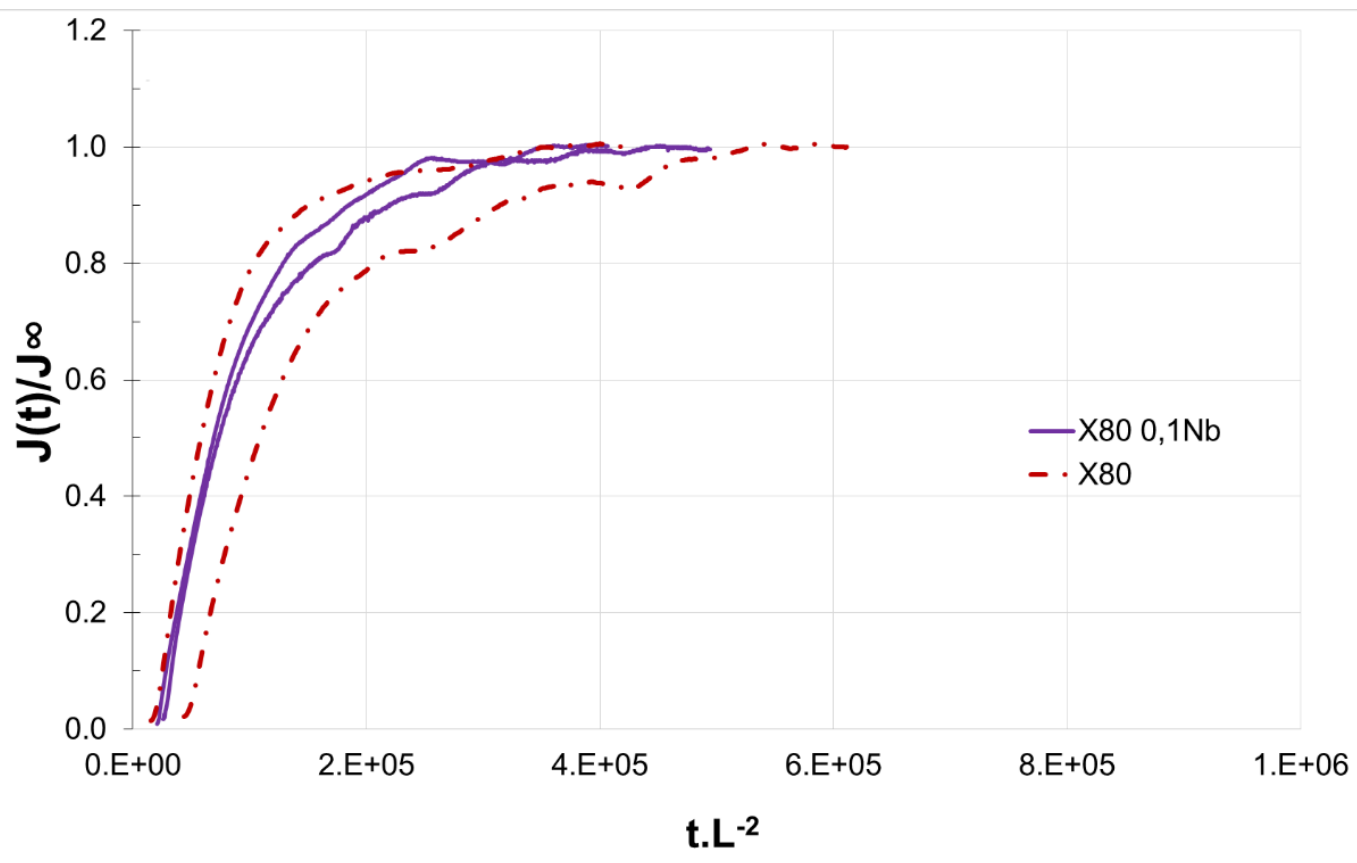

Figura 8. Curvas de permeabilidade de hidrogênio normalizadas.

Com o auxílio do programa OriginPro $8^{\circledR}$, foi possível a obtenção do ajuste das curvas de permeabilidade normalizadas com a segunda lei de Fick, cujo resultado, para a placa X80 0,1 Nb, está apresentado na Figura 9. O ajuste foi feito incluindo-se a equação mencionada ao banco de dados de ajustes não lineares do programa, com um valor de $\mathrm{n}$ igual a 6, conforme recomendado pela norma ASTM G148 [11]. Em seguida, iniciam-se as iterações de forma a alcançar os valores que melhor se aproximam dos resultados experimentais. Para dar início ao método iterativo, devese fornecer um valor inicial que neste caso foi de $1 \times 10^{-6} \mathrm{~cm}^{2} . \mathrm{s}^{-1}$.

As curvas obtidas através da implementação do modelamento teórico no programa apresentam um desvio em relação às curvas obtidas experimentalmente. Este desvio mostra a influência dos sítios de ancoramento na difusão do hidrogênio atômico pelo material. Os valores de tlag obtidos graficamente através das curvas experimentais de permeabilidade não são afetados por este afastamento, pois são obtidos a partir de $63 \%$ do tempo para atingir-se o estado estacionário, e nesta posição o ajuste com o modelo teórico é praticamente perfeito. Os valores de difusividade aparente obtidos através do método iterativo do programa OriginPro $8^{\circledR}$ e através do método tlag podem ser observados na Tabela 3. Nota-se que os valores obtidos são bem próximos, portanto o método de obtenção da difusividade através da obtenção gráfica do tlag mostra-se eficiente. 


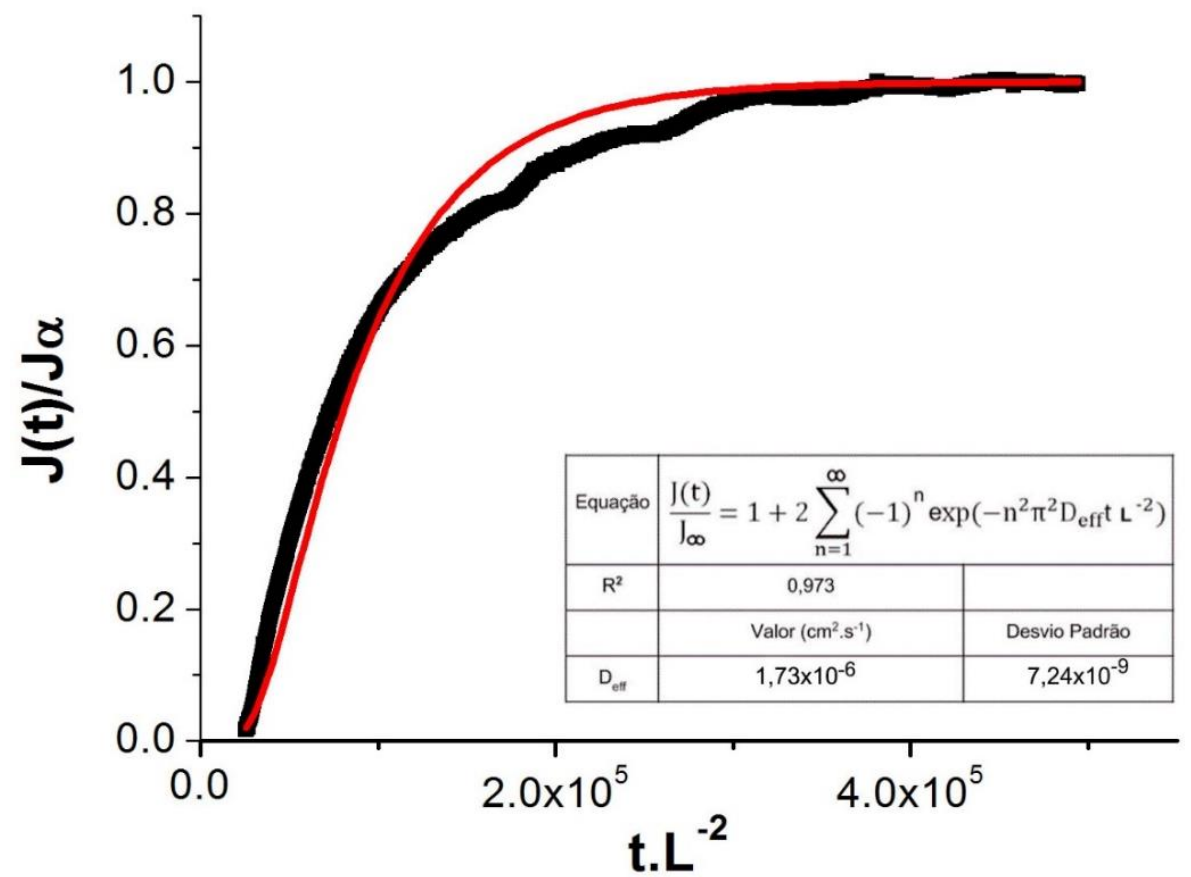

Figura 9. Ajuste realizado com o programa OriginPro $8^{\circledR}$ das curvas normalizadas (em preto) com o modelo teórico (em vermelho) para um dos ensaios da placa X80 0,1 Nb.

Tabela 3 Relação dos resultados obtidos através do método tlag e do método de ajuste

\begin{tabular}{|c|c|c|c|c|}
\cline { 2 - 5 } \multicolumn{1}{c|}{} & $\begin{array}{c}\text { Ajuste } \\
\text { Deff }\left(\mathrm{cm}^{2} \cdot \mathrm{s}^{-1}\right)\end{array}$ & $\begin{array}{c}\text { Desvio } \\
\text { padrão }\end{array}$ & $\mathrm{R}^{2}$ & $\begin{array}{c}\text { tlag } \\
D_{\text {eff }}\left(\mathrm{cm}^{2} . \mathrm{s}^{-1}\right)\end{array}$ \\
\hline \multirow{2}{*}{$\times 800,1 \mathrm{Nb}$} & $1,73 \times 10^{-6}$ & $7,24 \times 10^{-9}$ & 0,973 & $1,75 \times 10^{-6}$ \\
\cline { 2 - 5 } & $1,92 \times 10^{-6}$ & $5,82 \times 10^{-9}$ & 0,987 & $1,90 \times 10^{-6}$ \\
\hline \multirow{2}{*}{$\times 80$} & $1,18 \times 10^{-6}$ & $6,03 \times 10^{-9}$ & 0,971 & $1,23 \times 10^{-6}$ \\
\cline { 2 - 5 } & $2,30 \times 10^{-6}$ & $9,82 \times 10^{-9}$ & 0,986 & $2,27 \times 10^{-6}$ \\
\hline
\end{tabular}

A aplicação do ajuste conforme a segunda lei de Fick, através do programa OriginPro $8^{\circledR}$, comprovou os resultados obtidos pelo método tlag, sendo que este último é de mais fácil aplicação. Os parâmetros de permeabilidade foram praticamente iguais entre os dois métodos e mostraram que os dois materiais têm mesmo comportamento nessa propriedade. Essa igualdade de permeabilidade é atribuída ao fato de que os materiais apresentam as mesmas características microestruturais.

\section{CONCLUSÕES}

Os parâmetros de permeabilidade encontrados para a placa X80 0,1 Nb e tubo X80 são praticamente iguais, indicando que as diferenças na composição química dos materiais, principalmente os teores de $\mathrm{C}$ e Nb, não influenciaram esta propriedade de modo significativo.

A semelhança no comportamento dos parâmetros de permeabilidade dos materiais foi devida às similaridades nas suas microestruturas, ambas com matriz ferrítica e microconstituinte $\mathrm{M} / \mathrm{A}$. Este trabalho evidencia que o tipo e quantidade de traps em um material são determinados pela microestrutura.

O afastamento observado nas curvas normalizadas em relação às curvas experimentais não afeta a validade do método tlag, pois os dados obtidos 
graficamente para os tratamentos matemáticos deste método são obtidos da região onde o ajuste com o modelo teórico é praticamente perfeito.

Os valores de difusividade aparente encontrados pelo método tlag são próximos aos encontrados através do ajuste pelo modelo teórico e programa OriginPro 8®, indicando que o método tlag é eficiente, além de vantajoso, por apresentar maior praticidade para obtenção de parâmetros de permeabilidade válidos.

\section{Agradecimentos}

Os autores agradecem à CBMM pelo apoio à pesquisa, através do projeto: "Pesquisa e Desenvolvimento de Aços ARBL", o qual é desenvolvido junto à FUSP.

\section{REFERÊNCIAS}

1 Zhou C, Zheng S, Chen C, Lu G. The effect of the partial pressure of $\mathrm{H}_{2} \mathrm{~S}$ on the permeation of hydrogen in low carbon pipeline steel. Corrosion Science. 2013; 67:184192.

2 Shionahara Y, Hara T. Metallurgical design of UOE linepipe for sour service. Proceedings of the Microalloyed Steels for Sour Service, International Seminar, Companhia Brasileira de Metalurgia e Mineração - CBMM. São Paulo, Brasil. Araxá. 2012.

3 Park GT, Koh SU, Jung HG, Kim KG. Effect of microstructure on the hydrogen trapping efficiency and hydrogen induced cracking of linepipe steel. Corrosion Science. 2008;50:1865-1871.

4 Huang F, Li XG, Liu J. Qu YM, Jia J, Du CW. Hydrogen-induced cracking susceptibility and hydrogen trapping efficiency of different microstructure X80 pipeline steel. Journal of Materials Science.2011;46:715-722.

5 Dong CF, Li XG, Liu ZY, Zhang YR. Hydrogen-induced cracking and healing behaviuor in X70 steel. Journal of Alloys and Compounds. 2009;484(1-2):966-972.

6 Xue HB, Cheng YF. Characterization of inclusions of X80 pipeline steel and its correlation with hydrogen-induced cracking. Corrosion Science. 2011;53(4):1201-1208.

7 Wang SH, Luu WC, Ho KF, Wu JK. Hydrogen permeation in a submerged arc weldment of TMCP steel. Materials Chemistry and Physics. 2003; 77(2):447-454.

8 Devanathan, MA.; Starchurski Z. The absorption and diffusion of electrolytic hydrogen in palladium. 1962;270(1340).

9 Gray JM. Technology of microalloyed steel for large diameter pipe, international journal of pressure vessels and piping. 1974; 2(2):95-122.

10 Cheng YF. Analysis of electrochemical hydrogen permeation through X65 pipeline steel and its implications on pipeline stress corrosion cracking. International Journal of Hydrogen Energy. 2007; 32(9):1269 1276.

11 ASTM Standard G148 97. Permeation, and Transport in Metals by an Electrochemical Technique. Standard Practice for Evaluation of Hydrogen Uptake. ASTM international; 2003. 